\title{
Influence of vacancies on the melting transition of hard disks in two dimensions
}

\author{
Martin A. Bates and Daan Frenkel \\ FOM Institute for Atomic and Molecular Physics, Kruislaan 407, 1098 SJ Amsterdam, The Netherlands
}

(Received 22 November 1999)

\begin{abstract}
We present the results of molecular dynamics simulations of two-dimensional (2D) hard disk systems in the vicinity of melting. The simulations are used to calculate the elastic constants, which can be used to estimate the location of the Kosterlitz-Thouless dislocation unbinding transition. Simulations on defect-free lattices indicate that this transition is expected to occur at essentially the same density as a first-order solid-isotropic transition and so it is not possible to rule out either a one step weak first-order transition between the solid and the isotropic fluid or a two step transition via a hexatic phase. Simulations performed on systems with vacancies indicate that the elastic constants are essentially unchanged at constant density. This result implies that vacancies have little influence on the melting of $2 \mathrm{D}$ hard disk solids.
\end{abstract}

PACS number(s): 62.20.Dc, 05.20.-y

\section{INTRODUCTION}

The nature of the melting transition in two-dimensional (2D) systems has long been a controversial problem. 2D solids are qualitatively different than higher-dimensional systems because the density-density correlation function decays algebraically to zero. This means that 2D solids possess only quasi-long-range positional order and not the true long-range positional order characteristic of three-dimensional (3D) solids [1]. In the early 1970s, Kosterlitz and Thouless (KT) suggested that the nature of the melting transition in $2 \mathrm{D}$ is qualitatively different than that in $3 \mathrm{D}$ and showed that this could proceed as a continuous transition, initiated by the dissociation of dislocation pairs [2]. It was later shown that the resulting phase is not isotropic since it retains quasi-longrange bond orientational order and that a second transition from this hexatic phase, via the formation of disclinations, is necessary for the system to become isotropic [3-5]. According to the Kosterlitz-Thouless-Halperin-Nelson-Young (KTHNY) theory, 2D solids melt via two successive continuous transitions involving the unbinding of dislocations and disclinations, respectively. The solid-hexatic dislocation unbinding transition is predicted to occur when the dimensionless combination of elastic constants $K$ equals $16 \pi[3,4]$,

$$
K=\left[\left(\frac{1}{\mu}+\frac{1}{\lambda+\mu}\right) \frac{k_{B} T}{4 a^{2}}\right]^{-1}=16 \pi,
$$

where $\lambda$ and $\mu$ are the 2D Lamé elastic constants and $a$ is the lattice spacing. However, the KTHNY theory predicts only the point at which the solid becomes unstable to the generation of free dislocations and does not rule out the possibility that this transition can be preempted by, for example, a single first-order transition between the solid and isotropic liquid $[6,7]$.

Many experimental [8-11] and simulation [12-23] studies have focussed attention on the determination of the melting pathway of 2D solids and these suggest that the melting scenario for 2D systems is not universal but is dependent on specific properties of the systems, that is, on the interparticle potential. The experimental evidence appears to point to a continuous pathway via the hexatic phase, whereas contrasting evidence for both continuous and first-order transitions has been provided by simulation studies. Indeed, if we concentrate on the most simple 2D system with translational degrees of freedom, namely hard disks, the situation is not at all clear. Simulation studies provide inconsistent results, with claims for a weak first-order solid-isotropic transition $[12,13,15,19,20]$ and also a continuous solid-isotropic transition [21]; however, since the simulations are performed on finite size systems, the possibility of the pressure tie line found for the first-order transition becoming shorter or vanishing altogether as the system size is increased cannot be ruled out [18]. The most recent study on extremely large systems appears to indicate that while a continuous solidisotropic transition does not occur, neither the first-order solid-isotropic pathway nor the continuous solid-hexaticisotropic pathway can be ruled out [23]. This appears to be due to the fact that the density at which the solid-hexatic dislocation unbinding transition is predicted to occur, that is, when $K$ reaches the value $16 \pi$, coincides with the density of the solid at which a first-order melting transition would take place [16]. While the question of whether the melting pathway for hard disks proceeds via a hexatic phase is unresolved, there is strong evidence for the existence of a hexatic phase in other 2D systems; for example, hard core disks with very narrow attractive (or repulsive) potentials, although this phase does not necessarily occur as part of the melting process [24]. Systems of particles interacting via such potentials exhibit an isostructural solid-solid transition in which the lattice spacing jumps discontinuously and, just as for a liquid-vapor transition, the isostructural transition can have a critical point above which the distinction between the two phases vanishes [25]. Since the bulk elastic constant $B=\lambda+\mu=\rho(\partial P / \partial \rho)_{T}$ vanishes at the critical point, there is a finite region around the critical point where $K<16 \pi$ within which the solid is unstable to dislocation unbinding. If the range of the potential is short enough so that the solid-solid critical point occurs far from the solid-liquid transition, the induced hexatic phase will also occur far from the melting density. In this case, another hexatic phase may also be involved in the melting transition. As the range of the potential is increased, the critical point shifts towards the melting line, the hexatic phase becomes considerably wider, and a firstorder hexatic-liquid transition can be observed [24,26]. The important result of these simulations is that small differences 
in the potential can drastically change the phase behavior and so experiments on colloidal systems [8-11], in which the interparticle potential is never truly hard body, and simulations of hard disk systems are not always strictly comparable.

Simulation studies into the melting transition of 2D hard disks have so far only been performed for perfect crystals. As the melting transition is approached defects are expected to occur in the solid. Speedy and Reiss [27] have studied the formation of cavities in 2D hard disk systems and have obtained estimates for the vacancy concentration in the solid phase. Their results indicate that at densities $\rho>0.98$ (units $\sigma^{-2}$, where $\sigma$ is the diameter of a disk), the vacancy concentration is small enough to be neglected. As the density is lowered, the vacancy concentration rapidly rises up to about two vacancies per 1000 particles at the melting density $\rho$ $\approx 0.91$. It is important to know whether vacancies at this concentration will influence the melting pathway and the major point of this paper is to investigate this. In Sec. II, we describe simulations performed on perfect crystalline systems, which are necessary as a reference system and, in Sec. III, the results of simulations on systems with vacancies are presented and discussed.

\section{SIMULATIONS ON PERFECT CRYSTAL SYSTEMS}

One of the most important implications of the KTHNY theory is that, at the solid-hexatic melting density, a certain combination of elastic constants $K$ takes on the universal value of $16 \pi$ [see Eq. (1)]. Since the elastic properties depend on the melting mechanism, the elastic constants should provide a useful tool for understanding the nature of the melting transition. We have determined the 2D Lamé elastic constants $\lambda$ and $\mu$ over a range of densities using molecular dynamics simulations on slightly distorted systems [28-30]. Both stretched and sheared box simulations were used to calculate $\lambda$ and $\mu$ using the stress-strain relations given by Wallace $[31,32]$. Care was taken to ensure that the applied strains were sufficiently small to give a linear response of the diagonal and off-diagonal components of the stress tensor, respectively, and that no plastic flow occured in the sheared box simulation. The bulk modulus was compared with that available directly from the equation of state, $B$ $=\rho(\partial P / \partial \rho)_{T}$, as a check for internal consistency. Once $\lambda$ and $\mu$ have been obtained as a function of density, it is straightforward to obtain $K$ [see Eq. (1)] and so determine the density at which the KT disclination unbinding transition should occur. Simulations to determine the elastic constants for the defect-free 2D hard disk solid have already been performed by Wojciechowski and Brańka [16] and by Sengupta et al. [33]. While there is a general agreement for the density dependence of the bulk elastic constant from these two studies (which can easily be checked using the equation of state), the values of the shear modulus obtained by Sengupta et al. are much lower than those by Wojciechowski and Brańka and the former authors claim that this large difference is caused by the small systems used by the latter [33]. Since the behavior of the perfect crystal is clearly important if we wish to understand systems with vacancies, we have performed extensive simulations to determine the density dependence of the elastic constants using systems of $N=200,480,1920$,
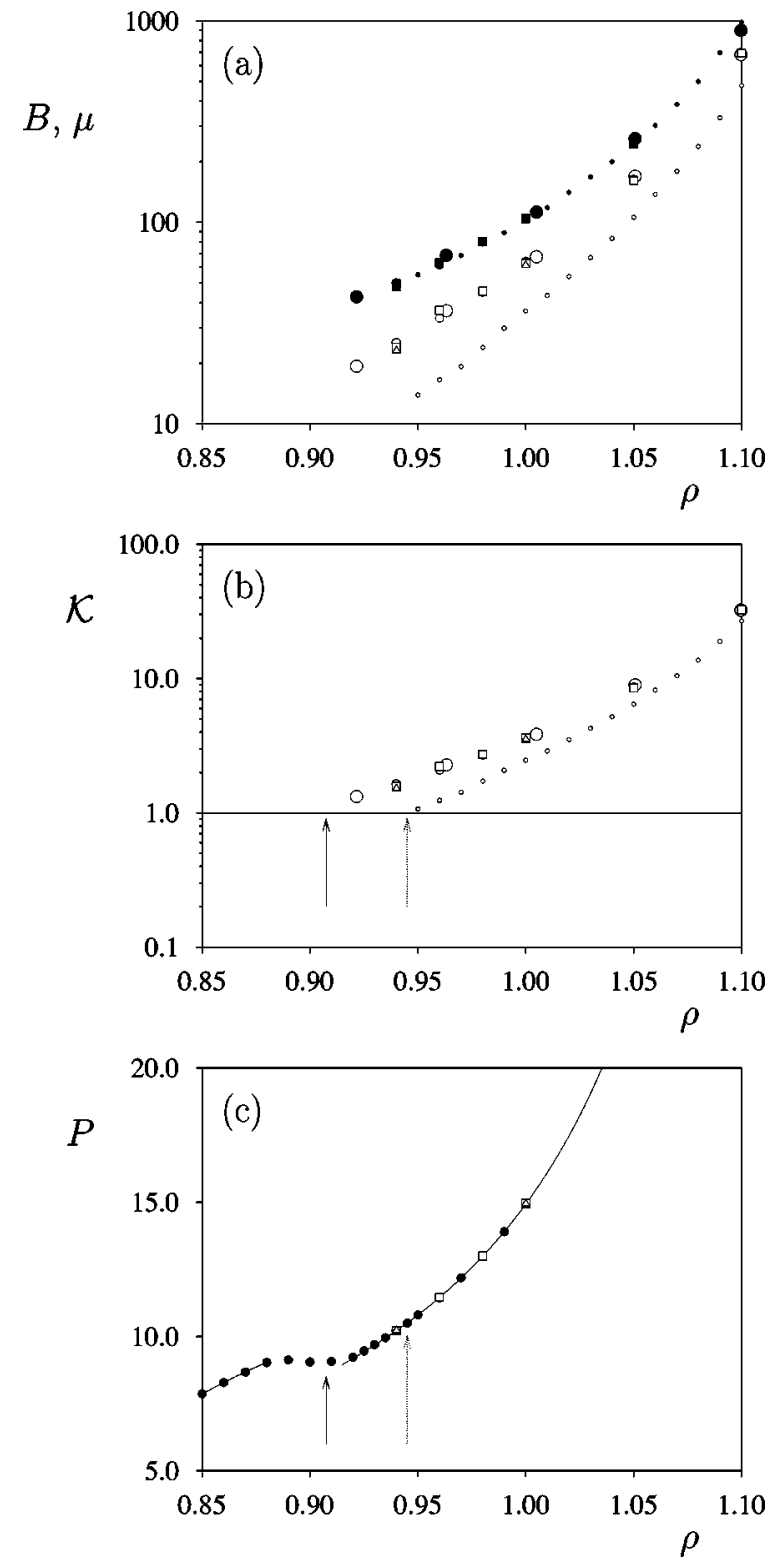

FIG. 1. (a) The bulk and shear elastic constants, $B=\lambda+\mu$ (filled symbols) and $\mu$ (open symbols), of the 2D hard disk solid obtained from simulations with $(\bigcirc) 480,(\square)$ 1920, and $(\triangle) 4320$ particles. ( $\bigcirc)$ Results of Wojciechowski and Brańka [16] and (०) Sengupta et al. [33]. (b) The dimensionless combination of elastic constants $\mathcal{K}=K / 16 \pi$ [see Eq. (1)]. (c) The equation of state obtained for a system of 1008 particles (filled symbols). The arrows indicate the location of the KT dislocation unbinding transition predicted by (solid) the present data and (dotted) Ref. [33]. Units: density $\rho$ in $\sigma^{-2}$, pressure $P$, and elastic constants $\lambda$ and $\mu$ in $k_{B} T / \sigma^{2}$.

and 4320 hard disks; the earlier simulations of small systems by Wojciechowski and Brańka were performed on 56 particles [16].

The density dependencies of the bulk and shear elastic constants, $B=\lambda+\mu$ and $\mu$, respectively, obtained from the stress-strain relations are plotted in Fig. 1, along with the density dependence of $K$ [see Eq. (1)] and the equation of state. The data for the bulk elastic constant $B$ are in essential agreement with earlier works. However, the data for the shear elastic constant $\mu$ for all system sizes studied appear to 


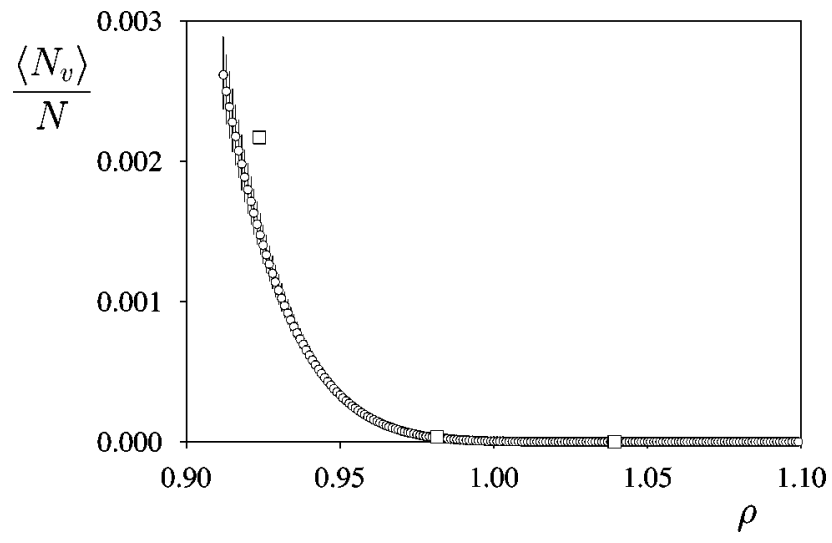

FIG. 2. Average number of vacancies $\left\langle N_{v}\right\rangle / N$ for the $2 \mathrm{D}$ hard disk solid. Data determined following the approach of Bennett and Alder (see text) [35] and ( $\square$ ) data obtained by Speedy and Reiss [27]. Units: density $\rho$ in $\sigma^{-2}$.

be in good agreement with that obtained by Wojciechowski and Brańka [16], rather than that of Sengupta et al. [33]. Indeed, the only significant difference between our results and those of Wojciechowski and Brańka is for the lowest density studied, $\rho=0.92$, where the pressure in the larger systems is found to be slightly higher than in the smaller ones, presumably because the periodic boundary conditions combined with the small box size helps stabilize the solid [23]. Indeed, at this density we were unable to obtain reliable results for the shear elastic constant because, even for very small strains, plastic flow occured in the simulation. The location of the KT dislocation unbinding transition, which occurs when $K=16 \pi$, determined from our simulations differs rather significantly from that determined from the results of Sengupta et al., as shown in Fig. 1(b). Figure 1(c) indicates the location of the KT transition in relation to the equation of state. The present results indicate that the transition should occur at $\rho=0.9075(25)$, essentially at the same density at which the first-order transition would occur $[15,17,18]$. We note that the elastic constants determined are the bare elastic constants and, as such, are an upper estimate of the elastic constants of the infinite size system and so provide a lower bound to the KT transition density. However, the results for the largest system appear to indicate that the renormalisation of the elastic constants by longer wavelength phonons is very weak. Moreover, if we allow for algebraic decay of $K$ in the vicinity of melting [4], then we again might expect the KT transition density to shift to very slightly higher densities. The results of Sengupta et al. lead to a much higher value of $\rho=0.9450$ (25) for the KT transition density. This seems somewhat surprising since it is rather deep into the region which is generally taken to be solid $[15,18]$.

We conclude this section by noting that analysis of the elastic constants of the 2D hard disk system indicates that the KT dislocation unbinding solid-hexatic transition occurs at $\rho=0.9075(25)$, that is, essentially at the same density at which a solid would melt via a first-order solid-isotropic transition [15]. It appears that we cannot say one way or the other whether the 2D hard disk solid melts discontinuously through a one stage first-order transition or continuously through a two stage transition via a hexatic phase. This result
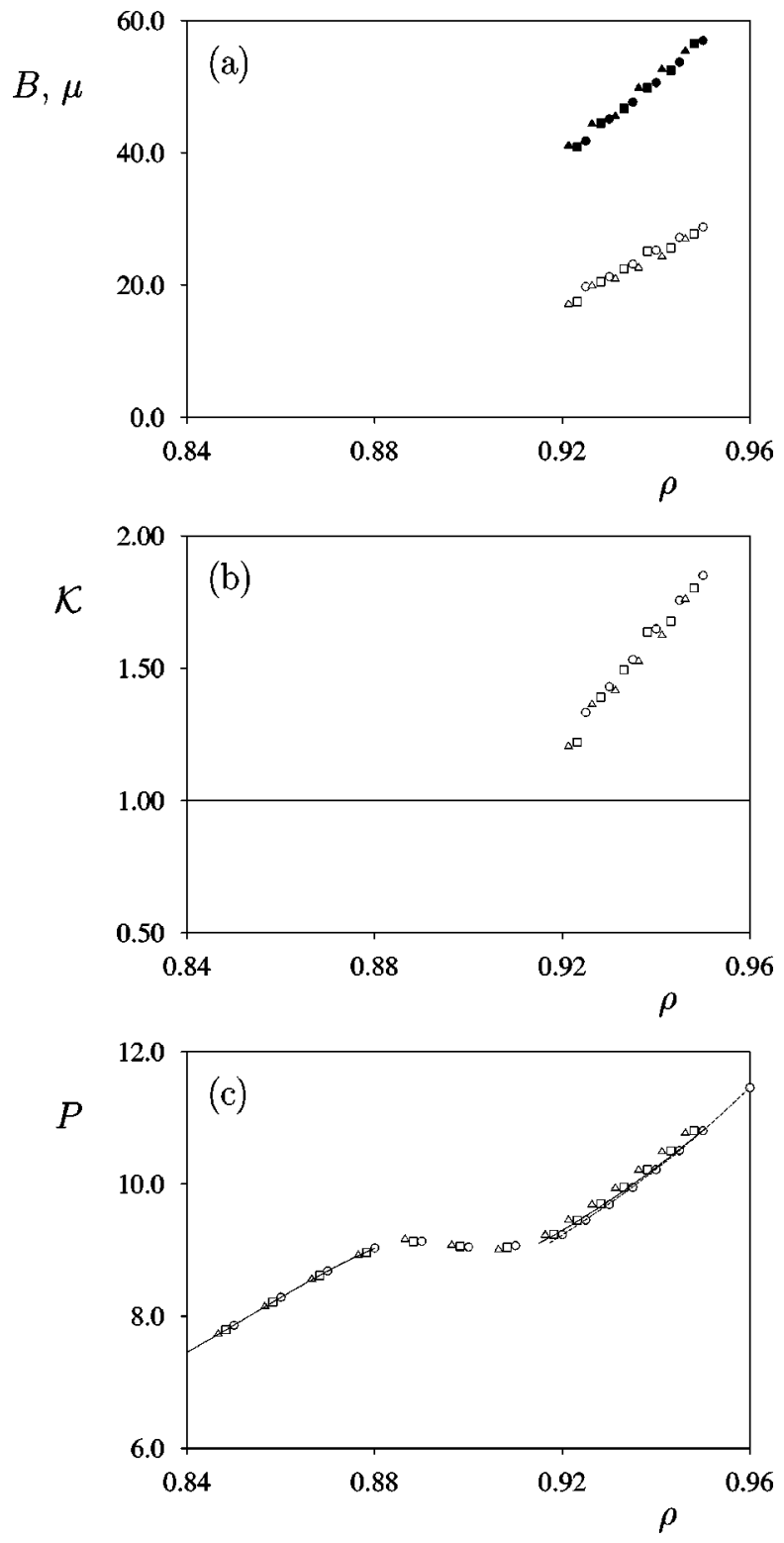

FIG. 3. (a) The bulk and shear elastic constants, $B=\lambda+\mu$ (filled symbols) and $\mu$ (open symbols), of the $2 \mathrm{D}$ hard disk solid with vacancies. Simulations were performed on a system with 1008 lattice sites and $(\bigcirc)$ zero vacancies, $(\square)$ two vacancies, and $(\triangle)$ four vacancies. (b) The dimensionless combination of elastic constants $\mathcal{K}=K / 16 \pi$ [see Eq. (1)]. (c) The equations of state obtained for the $2 \mathrm{D}$ hard disk solid with vacancies. The solid line indicates the equation of state taking vacancies into account, whereas the dotted line indicates the equation of state with no vacancies. Units: density $\rho$ in $\sigma^{-2}$, pressure $P$, and elastic constants $\lambda$ and $\mu$ in $k_{B} T / \sigma^{2}$.

is entirely consistent with large scale simulations of $2 \mathrm{D}$ hard disk systems [23].

\section{SIMULATIONS ON DEFECT CRYSTAL SYSTEMS}

We may expect that vacancies in a crystalline lattice will soften the system, leading to lower elastic constants and a shift of the KT transition to higher densities. To properly determine the influence of vacancies in the solid phase, we must of course ensure that the equilibrium concentration is 
achieved for each density studied. This is not an easy task in a simulation, because the number of lattice sites is usually a conserved quantity. Even if by some means the system can change the number of lattice sites, it will be at the cost of a distortion or a restriction of the number of lattice sites that can be achieved and so the solid cannot achieve its true equilibrium concentration of vacancies. An excellent discussion of this and other problems of simulating solids is given by Swope and Andersen [34]. To understand if vacancies influence the melting transition in 2D hard disks, we have taken a different approach in which we simulate a few systems in which the vacancy concentration is fixed, ranging from zero (the perfect crystal) up to about double the values determined by Speedy and Reiss at densities in the vicinity of the melting transition [27]. Before discussing the calculations for the elastic constants, we note that we have computed the vacancy concentration as a function of density, as an independent check for the values obtained by Speedy and Reiss. We have followed a method similar to that used by Bennett and Alder for hard spheres [35]; the general idea of this method is to measure the Gibbs free energy difference on the removal of a particle (or the creation of a vacancy). The Helmholtz free energy difference $f_{v}$ on the insertion of a particle into a vacancy can be calculated by trial insertion on a system with a single vacancy,

$$
f_{v}=-k_{B} T \log \left[V_{W S} P_{a c c}\left(V_{W S}\right)\right],
$$

where $V_{W S}$ is the volume of the Wigner-Seitz cell and $P_{a c c}\left(V_{W S}\right)$ is the probability that a trial insertion will be accepted within the vacant cell. However, since the product $V_{W S} P_{a c c}\left(V_{W S}\right)$ is equal to the cavity volume, actual particle insertions do not need to be performed, rather the cavity volume needs to be calculated. Note that we are assuming that interstitial-vacancy pairs are not formed (and thus that no new vacancies are created) and so the cavity volume measured is due only to the presence of the one imposed vacancy. Once the change in free energy of a crystal due to the creation of a vacancy, which is equal to $-f_{v}$, is known the average number of vacancies may be calculated [35],

$$
\frac{\left\langle N_{v}\right\rangle}{N}=\exp \left[-\left(\mu-f_{v}\right) / k_{B} T\right] .
$$

The cavity volume was calculated as a function of density in the solid phase using the algorithm described in Ref. [27]. For the chemical potential $\mu$ we have used the value determined at melting by Alder, Hoover, and Young [36] along with thermodynamic integration along the solid branch of the equation of state, which gives results consistent with those in Ref. [27]. The results for the vacancy concentration in 2D hard disk solids are shown in Fig. 2. We find fair agreement with the results of Speedy and Reiss at the points where they calculated the vacancy concentration.
Molecular dynamics simulations were performed for a defect-free system of $N=1008$ particles as in Sec. II, to obtain the equation of state and the elastic constants as a function of density. The same system, with $N_{\text {vac }}$ particles removed, was then used to investigate the influence of vacancies; we performed two series of simulations with $N_{v a c}=2$ and 4. Simulations were performed only for densities $\rho \leqslant 0.95$, where vacancies are predicted to occur at a high enough density such that they cannot be ignored [27]. In every case, long equilibration runs [50000 molecular dynamics (MD) steps or more, where one step means $N$ collisions) were performed to ensure that the vacancy had diffused away from its initial location. Results for the density dependencies of the elastic constants and pressure for the different systems with $N_{v a c}=0,2$, and 4 are shown in Fig. 3 . Note that in all cases, the density $\rho$ is the number density of the system. It is apparent from Figs. 3(a) and 3(b) that the elastic constants do not depend significantly on the vacancy concentration. Indeed, the location of the KT transition [see Fig. 3(b)] appears to be essentially unchanged when vacancies are introduced into the system. The only observable difference occurs in the equation of state [see Fig. 3(c)]. At number densities $\rho \leqslant 0.88$, the data for the three systems all follow the same continuous line, as we expect since the liquid is known to be the stable phase in this regime [18] and so the introduction of vacancies only serves to lower the density. This is not the case in the solid branch. Here, the introduction of vacancies leads to a shift in the equation of state to lower densities. Since we do not expect the crystalline system to be able to rearrange itself to form a system with fewer lattice sites [34], the data do not lie on the same curve. The pressure for each system is essentially constant at fixed lattice site density, which means that the pressure is increased on the introduction of vacancies at fixed number density [see Fig. 3(c)]. From the results presented in Fig. 2, we expect the system to follow an equation of state which crosses smoothly from that determined for $N_{v a c}=0$ to that for $N_{v a c}=2$, as shown in Fig. 3(c). While this means that the melting density of the solid will be reduced, this is not shifted significantly $(\sim 0.2 \%)$ for the vacancy concentrations typical of the hard disk fluid at melting [27]. We conclude that the melting mechanism is essentially unchanged with the introduction of the small number of vacancies expected in the vicinity of the melting transition.

\section{ACKNOWLEDGMENTS}

The work of the FOM Institute is part of the research program of FOM (Stichting Fundamenteel Onderzoek der Materie) and is supported by NWO (Nederlandse Organisatie voor Wetenschappelijk Onderzoek). We are grateful to B. M. Mulder and M. Noro for careful readings of the manuscript.
[1] K. Huang, Statistical Mechanics (Wiley, New York, 1963).

[2] J. M. Kosterlitz and D. J. Thouless, J. Phys. C 6, 1181 (1973); J. M. Kosterlitz, ibid. 7, 1046 (1974).

[3] B. I. Halperin and D. R. Nelson, Phys. Rev. Lett. 41, 121 (1978).
[4] D. R. Nelson and B. I. Halperin, Phys. Rev. B 19, 2457 (1979).

[5] A. P. Young, Phys. Rev. B 19, 1855 (1979).

[6] D. S. Fisher, B. I. Halperin, and R. Morf, Phys. Rev. B 20, 4692 (1979).

[7] S. T. Chui, Phys. Rev. B 28, 178 (1983). 
[8] C. A. Murray and D. H. Van Winkle, Phys. Rev. Lett. 58, 1200 (1987).

[9] R. E. Kusner, J. A. Mann, J. Kerins, and A. J. Dahm, Phys. Rev. Lett. 73, 3113 (1994).

[10] A. H. Marcus and S. A. Rice, Phys. Rev. Lett. 77, 2577 (1996).

[11] K. Zahn, R. Lenke, and G. Maret, Phys. Rev. Lett. 82, 2721 (1999).

[12] B. J. Alder and T. E. Wainwright, Phys. Rev. 127, 359 (1962).

[13] W. G. Hoover and F. H. Ree, J. Chem. Phys. 49, 3609 (1968).

[14] J. Q. Broughton, G. H. Gilmer, and J. D. Weeks, Phys. Rev. B 25, 4651 (1982).

[15] K. J. Strandburg, J. A. Zollweg, and G. V. Chester, Phys. Rev. B 30, 2755 (1984).

[16] K. W. Wojciechowski and A. C. Brańka, Phys. Lett. A 134, 314 (1988).

[17] J. A. Zollweg, G. V. Chester, and P. W. Leung, Phys. Rev. B 39, 9518 (1989).

[18] J. A. Zollweg and G. V. Chester, Phys. Rev. B 46, 11186 (1992).

[19] J. Lee and K. J. Strandburg, Phys. Rev. B 46, 11190 (1992).

[20] H. Weber and D. Marx, Europhys. Lett. 27, 593 (1994).

[21] J. F. Fernández, J. J. Alonso, and J. Stankiewicz, Phys. Rev. Lett. 75, 3477 (1995).
[22] K. Bagchi, H. C. Andersen, and W. Swope, Phys. Rev. E 53, 3794 (1996).

[23] A. Jaster, Phys. Rev. E 59, 2594 (1999).

[24] P. Bladon and D. Frenkel, Phys. Rev. Lett. 74, 2519 (1995).

[25] P. Bolhuis and D. Frenkel, Phys. Rev. Lett. 72, 2211 (1994).

[26] T. Chou and D. R. Nelson, Phys. Rev. E 53, 2560 (1996).

[27] R. J. Speedy and H. Reiss, Mol. Phys. 72, 1015 (1991).

[28] D. Frenkel and A. J. C. Ladd, Phys. Rev. Lett. 59, 1169 (1987).

[29] K. J. Runge and G. V. Chester, Phys. Rev. A 36, 4852 (1987).

[30] D. Frenkel, in Simple Molecular Systems at Very High Density, edited by A. Polian, P. Loubeyre, and N. Boccara (Plenum, New York, 1989).

[31] D. C. Wallace, Phys. Rev. 162, 776 (1967).

[32] D. C. Wallace, in Solid State Physics, edited by H. Ehrenreich, F. Seitz, and D. Turnbull (Academic, New York, 1970), Vol. 25.

[33] S. Sengupta, P. Nielaba, M. Rao, and K. Binder, Phys. Rev. E 61, 1072 (2000).

[34] W. C. Swope and H. C. Andersen, Phys. Rev. A 46, 4539 (1992).

[35] C. H. Bennett and B. J. Alder, J. Chem. Phys. 54, 4796 (1971).

[36] B. J. Alder, W. G. Hoover, and D. A. Young, J. Chem. Phys. 49, 3688 (1968). 